\title{
A Ground Fine Alignment of Strapdown INS under a Vibrating Base
}

\author{
Qian Li, Yueyang Ben, Zhongjun Zhu and Jiaolong Yang \\ (College of Automation, Harbin Engineering University, Harbin, China) \\ (E-mail: jay362033946@qq.com)
}

In order to solve the problem of a strapdown Inertial Navigation System (INS) under a vibrating base, a ground fine alignment method is proposed in this paper, and it is about reconstructing the error equations of a strapdown INS. In this method, the effects aroused by the linear and angular vibration are considered as system and measurement noise; in addition, they are also regarded as the components of a system matrix. This setting avoids the effects of linear and angular vibration under a vibrating base. The results of simulation and experiment show that our method is suitable for a ground fine alignment of a strapdown INS under a vibrating base.
KEY WORDS

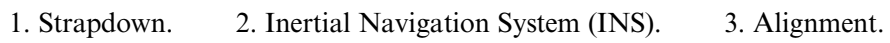

Submitted: 14 March 2012. Accepted: 9 June 2012. First published online: 29 August 2012.

1. INTRODUCTION. The purpose of fine alignment is to obtain an accurate initial strapdown attitude matrix between the body frame and navigation frame and, at the same time, set the misalignments to zero (Qin, 2006). The performance of strapdown Inertial Navigation System (INS) depends on the accuracy of the initial alignment, and the strapdown INS has to accomplish fine alignment before normal navigation and operation. Therefore the process of initial alignment, which requires high accuracy and speed, is of great importance in the operation of strapdown INS (Sun, 1996).

Under a vibrating base, (e.g., with a ground vehicle whose engine is running, or an aircraft which is preparing to take off), the strapdown INS alignment is likely to be disturbed by linear and angular vibrations. Therefore, the strapdown INS has to withstand the vibrations which include pitch, roll and yaw; of course, some other movements such as heave, surge and sway may take place as well (Silson, 2011). This means that extra disturbed accelerations and angular velocities may occur during the alignment process. Conventionally, alignment methods with a gyrocompass loop and Kalman filter are frequently used. It is known that the gyrocompass loop method can 
only find North with the gyrocompass effect when the vehicle is operating properly without linear vibration (Dai et al., 2011); so, any extra disturbed accelerations would make this method feeble under vibrating conditions. The other method, using a standard Kalman filter, utilizes modern control theory to estimate the misalignment angles, and is feasible for linear and angular vibrations (Qin, 2006). However, the disturbed acceleration and angular velocities may affect the estimation process (Vasconcelos et al., 2011; Qin, 2005), so that the estimation duration would be extended. Also, the ground vehicle then has to wait for the long-term alignment before entering into the normal operating condition.

In order to shorten the ground fine alignment time, we propose a ground fine alignment method in which the linear and angular vibration are considered as system and measurement noises as a result of avoiding their effects on alignment. All the analysis, results of simulations and experiments confirm our method.

2. NOMENCLATURE. Nomenclatures used in this paper are:

$i \quad$ Inertial frame

e $\quad$ Earth frame

$b \quad$ Body frame

$n \quad$ Navigation frame

n' Computed navigation frame

L Latitude

$R \quad$ Earth radius

$f \quad$ Specific force

$V \quad$ Vehicle velocity

$\varphi \quad$ Misalignment between $n$ frame and $n$ ' frame

$\omega_{i e} \quad$ Angular rate of Earth rotation

$\nabla \quad$ Accelerometer bias

$\varepsilon \quad$ Gyro drift

$\delta x \quad$ Error of the term $x$

3. STRAPDOWN INS ERROR EQUATIONS. To accomplish the ground fine alignment, error equations of a strapdown INS should be used. In this paper, a local level North-East-Down frame is defined as the $n$ frame. All the strapdown INS error equations are projected on the $n$ frame.

After deriving the coarse alignment results and linearizing the strapdown INS error equation, we could obtain the linear and simple error equation. Referred to (Qin, 2005), the equation of velocity error $\delta V$ is:

$$
\delta \dot{\boldsymbol{V}}^{n}=\boldsymbol{f}^{n} \times \boldsymbol{\varphi}^{n}+\left(2 \boldsymbol{\omega}_{i e}^{n}+\boldsymbol{\omega}_{e n}^{n}\right) \times \delta \boldsymbol{V}^{n}+\nabla^{n}
$$

where:

superscript denotes the frame on which the vector is projected.

$\omega_{e n}$ is the angular velocity from the $n$ frame to the $e$ frame.

$\omega_{i e}$ is the angular velocity from the $e$ frame to the $i$ frame. 
The equation of attitude error is in the $\varphi$ formulation:

$$
\dot{\boldsymbol{\varphi}}^{n}=\boldsymbol{\varphi}^{n} \times \boldsymbol{\omega}_{i n}^{n}+\delta \boldsymbol{\omega}_{e n}^{n}+\varepsilon^{n}
$$

where $\omega_{i n}$ is the angular velocity from the $n$ frame to the $i$ frame.

This equation is generally applicable for any three-axis navigation frame mechanization.

The gyro drift and accelerator bias here are considered as constant terms. Since gyros and accelerators are mounted on the three-axes of the $b$ frame, we need to transform the gyro drift and accelerator bias from the $b$ frame to the $n$ frame.

$$
\varepsilon^{n}=C_{b}^{n} \varepsilon^{b}, \nabla^{n}=C_{b}^{n} \nabla^{b}
$$

We usually use the gyro drifts and accelerator biases as extended states of the strapdown INS model.

4. CONVENTIONAL GROUND Fine ALIGNMENT. Under a vibrating base, the strapdown INS of the vehicle would sense linear and angular vibrations, which would affect the ground fine alignment process as well. As a result, we chose in-motion ground fine alignment to accomplish the fine alignment task instead of stationary ground fine alignment.

In terms of Section 3, the strapdown INS error model which is suitable for inmotion and a vibrating base can be regarded as a linear system augmented with white noises. The state equation is represented as (Fang and Yang, 2011):

$$
\dot{\boldsymbol{X}}(t)=\boldsymbol{A}(t) \boldsymbol{X}(t)+\boldsymbol{B}(t) \boldsymbol{W}(t)
$$

where the state vector and state noise vector are:

$$
X(t)=\left[\begin{array}{llllllllll}
\delta V_{E} & \delta V_{N} & \varphi_{E} & \varphi_{N} & \varphi_{U} & \nabla_{x} & \nabla_{y} & \varepsilon_{x} & \varepsilon_{y} & \varepsilon_{z}
\end{array}\right]^{T}
$$

The subscripts $E, N$ and $U$ denote the East, West and Up direction of $n$ frame respectively, and the subscripts $x, y$ and $z$ denote the $\mathrm{x}, \mathrm{y}$ and $\mathrm{z}$ axis of $b$ frame respectively.

In Equation (4), $\boldsymbol{W}(t)$ is white noise vector and represents the system model noise, and the state matrix is:

$$
\boldsymbol{A}(t)=\left[\begin{array}{cc}
\boldsymbol{F}_{5 \times 5} & \boldsymbol{T}_{5 \times 5} \\
0_{5 \times 5} & 0_{5 \times 5}
\end{array}\right]
$$

where:

$$
\boldsymbol{F}_{5 \times 5}=\left[\begin{array}{cc}
\boldsymbol{F}_{2 \times 2} & \boldsymbol{F}_{2 \times 3} \\
0_{3 \times 2} & \boldsymbol{F}_{3 \times 3}
\end{array}\right]
$$

in which the following definitions apply:

$$
\boldsymbol{F}_{2 \times 2}=\left[\begin{array}{cc}
0 & 2 \omega_{i e} \sin L+\frac{V_{E} \tan L}{R} \\
-2\left(\omega_{i e} \sin L+\frac{V_{E} \tan L}{R}\right) & 0
\end{array}\right]
$$




$$
\begin{gathered}
\boldsymbol{F}_{2 \times 3}=\left[\begin{array}{ccc}
0 & -f_{U} & f_{N} \\
f_{U} & 0 & -f_{E}
\end{array}\right], \quad \boldsymbol{F}_{3 \times 2}=\left[\begin{array}{cc}
0 & -\frac{1}{R} \\
\frac{1}{R} & 0 \\
\frac{\tan L}{R} & 0
\end{array}\right] \\
\boldsymbol{F}_{3 \times 3}=\left[\begin{array}{ccc}
0 & \omega_{i e} \sin L+\frac{V_{E} \tan L}{R} & -\left(\omega_{i e} \cos L+\frac{V_{E}}{R}\right) \\
-\left(\omega_{i e} \sin L+\frac{V_{E} \tan L}{R}\right) & 0 & -\frac{V_{N}}{R} \\
\omega_{i e} \cos L+\frac{V_{E}}{R} & \frac{V_{N}}{R}
\end{array}\right]
\end{gathered}
$$

Note that the scale errors as well as the installation errors of gyro and accelerometer are eliminated by laboratory calibration. In addition, vertical velocity error is ignored in ground alignment for terrestrial application.

The system noise matrix for ground fine alignment is:

$$
\boldsymbol{B}(t)=\left[\begin{array}{ll}
\boldsymbol{T}_{5 \times 5} & 0_{5 \times 5} \\
0_{5 \times 5} & 0_{5 \times 5}
\end{array}\right]
$$

Assuming the strapdown attitude matrix is:

$$
C_{b}^{n}=\left[\begin{array}{lll}
C_{11} & C_{12} & C_{13} \\
C_{21} & C_{22} & C_{23} \\
C_{31} & C_{32} & C_{33}
\end{array}\right]
$$

we can obtain:

$$
\boldsymbol{T}_{5 \times 5}=\left[\begin{array}{ccccc}
C_{11} & C_{12} & 0 & 0 & 0 \\
C_{21} & C_{22} & 0 & 0 & 0 \\
0 & 0 & C_{11} & C_{12} & C_{13} \\
0 & 0 & C_{21} & C_{22} & C_{23} \\
0 & 0 & C_{31} & C_{32} & C_{33}
\end{array}\right]
$$

During in-motion alignment, the velocity differences between the strapdown INS and referenced system along the horizontal direction are defined as measurements. The reference system which provides the accurate velocity is usually either a Global Position System (GPS) or a Doppler Velocity Log (DVL) (Huang et al., 2010). As for the ground fine alignment, we consider the true velocity as zero and do not need a reference system i.e., the output horizontal velocities of strapdown INS compose measurements $\boldsymbol{Z}(t)$.

The measurement equation is:

$$
\boldsymbol{Z}(t)=\boldsymbol{H}(t) \boldsymbol{X}(t)+\boldsymbol{M}(t)
$$

where the measurement matrix is:

$$
\boldsymbol{H}(t)=\left[\begin{array}{llllllllll}
1 & 0 & 0 & 0 & 0 & 0 & 0 & 0 & 0 & 0 \\
0 & 1 & 0 & 0 & 0 & 0 & 0 & 0 & 0 & 0
\end{array}\right]
$$


and where $\boldsymbol{M}(t)$ represents the measurement noise vector, including the effects of measurement instruments and the disturbed accelerations on measurements.

In terms of the constructed state equation and measurement equations above, the discrete Kalman filter can be utilized to estimate the state vector in Equation (5). After deriving the convergence values of misalignments between navigation frame and computed navigation frame, we can correct the coarse strapdown attitude matrix, and then accomplish ground fine alignment (Song et al., 2010).

5. PROPOSED GROUND FINE ALIGNMENT. Unlike the normal stationary fine alignment, linear and angular vibrations raised by vehicle motor running would affect the alignment process.

In the deduced state equation and measurement equations, several terms could be disturbed by linear and angular vibrations. For instance, the specific force projected on the navigation frame in Equation (9), the East and North velocities in Equation (10) and the strapdown attitude matrix in Equation (10) vary as sine form since linear and angular vibrations exist. All variations make the Signal to Noise Ratio (SNR) lower than normal stationary alignment, so the time of alignment is extended (Jiang and Yu, 1992; Burak et al., 2004).

In order to solve this problem, we have to reconstruct the equations, and parameter identification technology has also to be employed.

5.1. Reconstruction of Error Equations. Since the $n$ frame has no movements relative to the $e$ frame under a vibrating base, the followed assumptions are valid:

$$
\boldsymbol{\omega}_{i n}^{n}=\boldsymbol{\omega}_{i e}^{n}, \delta \boldsymbol{\omega}_{e n}^{n}=\left[\begin{array}{lll}
0 & 0 & 0
\end{array}\right]^{T}
$$

and gyro drifts projected on the $n$ frame could be:

$$
\boldsymbol{\varepsilon}^{n}=\left(\overline{\boldsymbol{C}}_{b}^{n}+\Delta \boldsymbol{C}_{b}^{n}\right) \boldsymbol{\varepsilon}^{b}
$$

where:

$\overline{\boldsymbol{C}}_{b}^{n}$ is the constant part of the strapdown attitude matrix, i.e., the strapdown attitude matrix as the vehicle engine does not run.

$\Delta \boldsymbol{C}_{b}^{n}$ is the variational part of strapdown attitude matrix raised by angular vibration as the vehicle engine runs.

Further deduction yields:

$$
\varepsilon^{n}=\vec{\varepsilon}^{n}+\Delta \varepsilon^{n}
$$

During fine alignment, the gyro drifts along the axis of $b$ frame $\varepsilon^{b}$ is considered as constant vector, thus $\overline{\boldsymbol{\varepsilon}}^{n}$ is constant vector too.

Under a vibrating base, the amplitudes of angular vibrations are so small that we can make the following approximation:

$$
\Delta \boldsymbol{C}_{b}^{n} \approx\left[\begin{array}{ccc}
0 & -\Delta \text { Yaw } & \Delta \text { Roll } \\
\Delta \text { Yaw } & 0 & -\Delta \text { Pitch } \\
-\Delta \text { Roll } & \Delta \text { Pitch } & 0
\end{array}\right]
$$


Since the variations of angular vibrations $\Delta Y a w, \Delta$ Roll and $\Delta$ Pitch are periodic in sine form, $\Delta \boldsymbol{C}_{b}^{n}$ is periodic in sine form too:

$$
\Delta \varepsilon^{n}=\Delta C_{b}^{n} \varepsilon^{b}
$$

As a result, under a vibrating base, $\Delta \varepsilon^{n}$ varies periodically with the same frequency as the vehicle vibration.

Returning to Equation (2) and considering the characters under vibrating base, Equation (2) becomes:

$$
\dot{\varphi}^{n}=\varphi^{n} \times \omega_{i e}^{n}+\vec{\varepsilon}^{n}+\Delta \varepsilon^{n}
$$

Rewriting Equation (20) in matrix form yields:

$$
\left[\begin{array}{c}
\dot{\varphi}_{E} \\
\dot{\varphi}_{N} \\
\dot{\varphi}_{U}
\end{array}\right]=\left[\begin{array}{ccc}
0 & \omega_{i e} \sin L & -\omega_{i e} \cos L \\
-\omega_{i e} \sin L & 0 & 0 \\
\omega_{i e} \cos L & 0 & 0
\end{array}\right]\left[\begin{array}{c}
\varphi_{E} \\
\varphi_{N} \\
\varphi_{U}
\end{array}\right]+\left[\begin{array}{c}
\bar{\varepsilon}_{E} \\
\bar{\varepsilon}_{N} \\
\bar{\varepsilon}_{U}
\end{array}\right]+\left[\begin{array}{c}
\Delta \varepsilon_{E} \\
\Delta \varepsilon_{N} \\
\Delta \varepsilon_{U}
\end{array}\right]
$$

For the purpose of solving Equation (21), we make:

$$
\boldsymbol{A}=\left[\begin{array}{ccc}
0 & \omega_{i e} \sin L & -\omega_{i e} \cos L \\
-\omega_{i e} \sin L & 0 & 0 \\
\omega_{i e} \cos L & 0 & 0
\end{array}\right]
$$

and:

$$
\boldsymbol{X}=\left[\begin{array}{l}
\varphi_{E}-\varphi_{E_{0}} \\
\varphi_{N}-\varphi_{N_{0}} \\
\varphi_{U}-\varphi_{U_{0}}
\end{array}\right]
$$

The initial values of misalignments are $\varphi_{0}=\left[\varphi_{E 0}, \varphi_{N 0}, \varphi_{U 0}\right]^{T}$. Then Equation (21) could be expressed as:

$$
\dot{X}(t)=A X(t)+A \phi_{0}+\bar{\varepsilon}^{n}+\Delta \varepsilon^{n}
$$

by making the assumption:

$$
\boldsymbol{u}=A \varphi_{0}+\overrightarrow{\boldsymbol{\varepsilon}}^{n}
$$

which represents three equations:

$$
\begin{aligned}
& u_{E}=\varphi_{N_{0}} \omega_{i e} \sin L-\varphi_{U_{0}} \omega_{i e} \cos L+\varepsilon_{E} \\
& u_{N}=-\varphi_{E_{0}} \omega_{i e} \sin L+\varepsilon_{N} \\
& u_{U}=\varphi_{E_{0}} \omega_{i e} \cos L+\varepsilon_{U}
\end{aligned}
$$

we have:

$$
\dot{\boldsymbol{X}}(t)=\boldsymbol{A} \boldsymbol{X}(t)+\boldsymbol{u}+\Delta \boldsymbol{\varepsilon}^{n}
$$

Equation (25) with constant stimulation and periodic stimulation can be solved by the Laplace transformation. The Laplace transformation analytical method requires the determination of the characteristic values of Equation (25). Reduction of the determinant in (25) yields the characteristic of Equation (26).

$$
\boldsymbol{X}(s)=(s \boldsymbol{I}-\boldsymbol{A})^{-1}\left[\frac{\boldsymbol{u}}{s}-\Delta \varepsilon^{n}(s)\right]
$$


furthermore, assuming that:

$$
(s \boldsymbol{I}-\boldsymbol{A})^{-1}=\boldsymbol{D}
$$

we have:

$$
\begin{array}{ccc}
D_{11}=\frac{s}{s^{2}+\omega_{i e}^{2}}, & D_{12}=\frac{\omega_{i e} \sin L}{s^{2}+\omega_{i e}^{2}}, & D_{13}=-\frac{\omega_{i e} \cos L}{s^{2}+\omega_{i e}^{2}} \\
D_{21}=-\frac{\omega_{i e} \sin L}{s^{2}+\omega_{i e}^{2}}, & D_{22}=\frac{s^{2}+\omega_{i e}^{2} \cos ^{2} L}{s\left(s^{2}+\omega_{i e}^{2}\right)}, & D_{23}=\frac{\omega_{i e}^{2} \sin L \cos L}{s\left(s^{2}+\omega_{i e}^{2}\right)} \\
D_{31}=\frac{\omega_{i e} \cos L}{s^{2}+\omega_{i e}^{2}}, & D_{32}=\frac{\omega_{i e}^{2} \sin L \cos L}{s\left(s^{2}+\omega_{i e}^{2}\right)}, & D_{33}=\frac{s^{2}+\omega_{i e}^{2} \sin ^{2} L}{s\left(s^{2}+\omega_{i e}^{2}\right)}
\end{array}
$$

Referring to the Cramer rule, we inverse $\boldsymbol{D}$ back into the time domain:

$$
\boldsymbol{B}=\ell^{-1}\{\boldsymbol{D}\}
$$

then:

$$
\begin{array}{ccc}
B_{11}=\cos \omega_{i e} t & B_{21}=\sin L \sin \omega_{i e} t & B_{31}=-\cos L \sin \omega_{i e} t \\
B_{21}=-\sin L \sin \omega_{i e} t & B_{22}=\cos ^{2} L+\sin ^{2} L \cos \omega_{i e} t & B_{23}=\sin L \cos L\left(1-\cos \omega_{i e} t\right) \\
B_{31}=\cos L \sin \omega_{i e} t & B_{32}=\sin L \cos L\left(1-\cos \omega_{i e} t\right) & B_{33}=\sin ^{2} L+\cos ^{2} L \cos \omega_{i e} t
\end{array}
$$

Since the time of alignment under a vibration base is usually less than 20 minutes, and $\left|\omega_{i e} t\right|$ is less than 5 degrees, we make the following approximation:

$$
\cos \omega_{\text {ie }} t \approx 1, \sin \omega_{\text {ie }} t \approx \omega_{\text {ie }} t
$$

with Equation (30), Equation (29) becomes:

$$
\boldsymbol{B}(t)=\left[\begin{array}{ccc}
1 & t \omega_{i e} \sin L & -t \omega_{i e} \cos L \\
-t \omega_{i e} \sin L & 1 & 0 \\
t \omega_{i e} \cos L & 0 & 1
\end{array}\right]
$$

The next step is inversing Equation (26) back into the time domain:

$$
\boldsymbol{X}(t)=\int\left[\boldsymbol{B}(t) * \boldsymbol{u}-\boldsymbol{B}(t) * \Delta \varepsilon^{n}\right] d t
$$

Under a vibrating base, $\Delta \varepsilon^{n}$ varies periodically with the same frequency as the vehicle vibration. Therefore, the integration term $\int\left[\boldsymbol{B}(t)^{*} \Delta \varepsilon^{n}\right] d t$ is nearly equal to be zero. Equation (31) can be simplified as:

$$
\boldsymbol{X}(t)=\int[\boldsymbol{B}(t) * \boldsymbol{u}] d t
$$

Expansion of Equation (32) gives:

$$
\boldsymbol{X}(t)=\left[\begin{array}{c}
u_{E} t+\frac{t^{2}}{2} u_{N} \omega_{i e} \sin L-\frac{t^{2}}{2} u_{U} \omega_{i e} \cos L \\
-\frac{t^{2}}{2} u_{E} \omega_{i e} \sin L+u_{N} t \\
\frac{t^{2}}{2} u_{E} \omega_{i e} \cos L+u_{U} t
\end{array}\right]
$$


which represents the three equations:

$$
\boldsymbol{f}^{n}=\left[\begin{array}{c}
f_{d E}+\nabla_{E} \\
f_{d N}+\nabla_{N} \\
f_{d U}+\nabla_{U}+g
\end{array}\right]
$$

Referred to the computed navigation frame, the specific force by accelerometer sensing is:

$$
\boldsymbol{f}^{n^{\prime}}=\left[\begin{array}{ccc}
1 & \varphi_{U} & -\varphi_{N} \\
-\varphi_{U} & 1 & \varphi_{E} \\
\varphi_{N} & -\varphi_{E} & 1
\end{array}\right]\left[\begin{array}{c}
f_{d E}+\nabla_{E} \\
f_{d N}+\nabla_{N} \\
f_{d U}+\nabla_{U}+g
\end{array}\right]
$$

Expanding Equation (35) and ignoring the two-order small error quantities, we obtain:

$$
\begin{aligned}
& f_{E}^{n^{\prime}}=-g \varphi_{N}+f_{d E}+\nabla_{E} \\
& f_{N}^{n^{\prime}}=-g \varphi_{E}+f_{d N}+\nabla_{N} \\
& f_{U}^{n^{\prime}}=g+f_{d U}+\nabla_{U}
\end{aligned}
$$

From Equation (36), the misalignments coupled with gravitational acceleration act on the horizontal specific forces. So we should choose the horizontal velocity error as measurements for the Kalman filter.

Substituting Equation (24) into the first and second equations of Equation (36), and then making the integration in $[0, t]$, we can obtain:

$$
\begin{aligned}
& \Delta V_{E}(t)=\left(\nabla_{E}-g \varphi_{N 0}\right) t-\frac{t^{2}}{2} g u_{N}+\frac{t^{3}}{6} g \omega_{i e} u_{E} \sin L+V_{D E} \\
& \Delta V_{N}(t)=\left(\nabla_{N}+g \varphi_{E 0}\right) t+\frac{t^{2}}{2} g u_{E}+\frac{t^{3}}{6} g \omega_{i e}\left(u_{N} \sin L-u_{U} \cos L\right)+V_{D N}
\end{aligned}
$$

It can be seen from Equation (37) that the initial value of horizontal misalignments added by the accelerator bias construct the one-order term about time, and they could be estimated first. The terms $u_{N}$ and $u_{E}$ include real-time horizontal misalignments to construct the two-order term about time, and they could be estimated. Thus horizontal misalignments can be estimated quickly in our proposed method. But unfortunately, azimuth misalignment exists in $u_{E}$ and the three-order term about time $\frac{t^{3}}{6} g \omega_{i e}\left(u_{N} \sin L-u_{U} \cos L\right)$, so we need a longer duration to estimate it than horizontal ones. Moreover, the disturbed acceleration $f_{d}$ is integrated and since the disturbed acceleration is in a periodic form under a vibrating base:

$$
V_{D E} \approx V_{D E} \approx 0
$$

Making the denotations:

$$
\begin{aligned}
& \alpha_{1 E}=\left(\nabla_{E}-g \varphi_{N 0}\right), \alpha_{2 E}=-\frac{1}{2} g u_{N}, \alpha_{3 E}=\frac{1}{6} g \omega_{i e} u_{E} \sin L \\
& \alpha_{1 N}=\left(\nabla_{N}+g \varphi_{E 0}\right), \alpha_{2 N}=\frac{t^{2}}{2} g u_{E}, \alpha_{3 N}=\frac{1}{6} g \omega_{i e}\left(u_{N} \sin L-u_{U} \cos L\right)
\end{aligned}
$$


and rewriting Equation (37), Equation (39) may be derived:

$$
\begin{aligned}
& \Delta V_{E}(t)=\alpha_{1 E} t+\alpha_{2 E} t^{2}+\alpha_{3 E} t^{3}+V_{D E} \\
& \Delta V_{N}(t)=\alpha_{1 N} t+\alpha_{2 N} t^{2}+\alpha_{3 N} t^{3}+V_{D N}
\end{aligned}
$$

5.2. Filter Model. With Equation (39), the discrete form of estimating misalignments in our proposed method is:

$$
\left\{\begin{array}{l}
\boldsymbol{X}(k+1)=\boldsymbol{X}(k) \\
\Delta \boldsymbol{V}(k)=\boldsymbol{H}(k) \boldsymbol{X}(k)+\boldsymbol{V}_{D}(k)
\end{array}\right.
$$

The system state is:

$$
\boldsymbol{X}(k)=\left[\begin{array}{llllll}
\alpha_{1 E} & \alpha_{2 E} & \alpha_{3 E} & \alpha_{1 N} & \alpha_{2 N} & \alpha_{3 N}
\end{array}\right]^{T}
$$

The measurement is:

$$
\Delta \boldsymbol{V}(k)=\left[\begin{array}{c}
\Delta V_{E}(k) \\
\Delta V_{N}(k)
\end{array}\right]
$$

The disturbed term is:

$$
\boldsymbol{V}_{D}(k)=\left[\begin{array}{c}
V_{D E}(k) \\
V_{D N}(k)
\end{array}\right]
$$

The measurement matrix is:

$$
H(k)=\left[\begin{array}{llllll}
k T & (k T)^{2} & (k T)^{3} & 0 & 0 & 0 \\
0 & 0 & 0 & k T & (k T)^{2} & (k T)^{3}
\end{array}\right]
$$

where:

$k$ is the updating index.

$T$ is the updating interval of Kalman filter.

According to the well-known recurrent computation scheme of the Kalman filter, we estimate the states in Equation (41) with noise disturbance. With the horizontal velocity measurements under vibrating base, the Kalman filter works to estimate the state vector in Equation (41), and then we obtain misalignments in term of Equation (38).

$$
\begin{aligned}
\varphi_{E 0} & =\frac{\alpha_{1 N}}{g}-\frac{\nabla_{E}}{g} \\
\varphi_{N 0} & =-\frac{\alpha_{1 E}}{g}+\frac{\nabla_{N}}{g} \\
\varphi_{U 0} & =-\frac{\alpha_{1 E} \tan L}{g}-\frac{2 \alpha_{2 N}}{g \omega_{i e} \cos L}-\frac{\varepsilon_{E}}{\omega_{i e} \cos L}
\end{aligned}
$$

Without aided sensor instruments, such as rotation tables and GPS, we cannot calibrate instrument errors in the field, so instrument errors cannot be separated from misalignments. 


$$
\begin{aligned}
\delta \varphi_{E 0} & =\frac{\nabla_{E}}{g} \\
\delta \varphi_{N 0} & =-\frac{\nabla_{N}}{g} \\
\delta \varphi_{U 0} & =\frac{\varepsilon_{E}}{\omega_{i e} \cos L}
\end{aligned}
$$

So, we have to make the approximations:

$$
\begin{aligned}
& \varphi_{E 0} \approx \frac{\alpha_{1 N}}{g} \\
& \varphi_{N 0} \approx-\frac{\alpha_{1 E}}{g} \\
& \varphi_{U 0} \approx-\frac{\alpha_{1 E} \tan L}{g}-\frac{2 \alpha_{2 N}}{g \omega_{i e} \cos L}
\end{aligned}
$$

From Equation (46), it can be seen that, interestingly, our proposed method has the same alignment accuracy as the conventional stationary alignment methods.

\section{SIMULATION AND EXPERIMENT}

6.1. Simulation. Under the vibrating condition, the proposed ground fine alignment is tested. Gyro and accelerator outputs are generated by the strapdown INS simulator. The instrument measurement unit (IMU) errors are set as:

- The gyro constant drift: $0 \cdot 01 \%$

- The gyro measurement noise: $0.005^{\circ} / \sqrt{h}$

- The accelerator bias: $1 \times 10^{-4} \mathrm{~g}$

- The accelerator measurement noise: $1 \times 10^{-5} \mathrm{~g}$

Under the vibrating condition, the vehicle undertakes angular and linear vibrations. In angular vibration, the yaw $\psi$, the pitch $\theta$ and the roll $\gamma$ are controlled as:

$$
\begin{aligned}
\psi & =0.6^{\circ} \cos \left(2 \pi * 15 t+\rho_{1}\right) \\
\theta & =0.8^{\circ} \cos \left(2 \pi * 15 t+\rho_{2}\right) \\
\gamma & =0.3^{\circ} \cos \left(2 \pi * 15 t+\rho_{3}\right)
\end{aligned}
$$

in which $\rho_{1}, \rho_{2}$ and $\rho_{3}$ are angular vibration phases which are distributed randomly between 0 and $2 \pi$.

In the lineal vibration, the vehicle lineal vibration velocities are:

$$
V_{D i}=A_{D i} \cos \left(\frac{2 \pi}{T_{D i}} t+\gamma_{D i}\right)
$$

where:

$i=E, N, U$

$\gamma_{D i}$ are lineal vibration phases which obey the uniform distribution on the interval between 0 and $2 \pi$. 


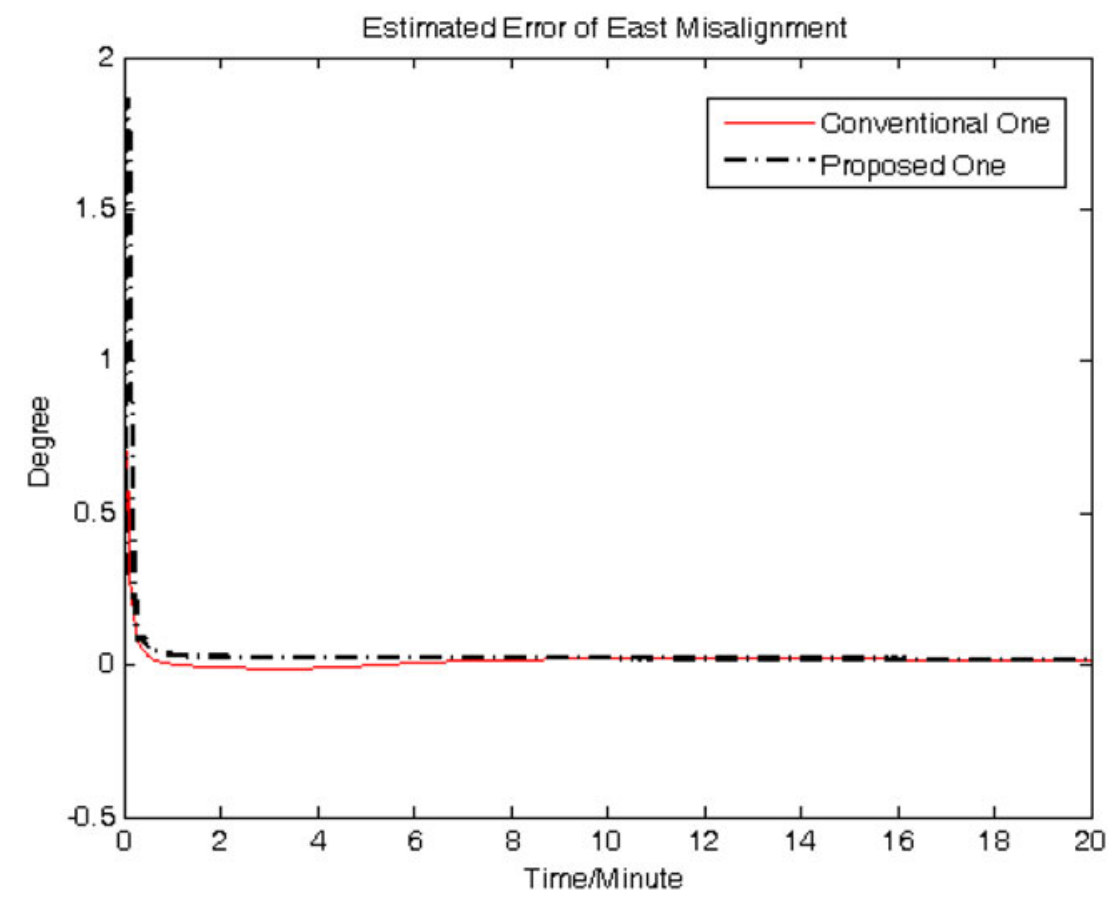

Figure 1. Estimated error of East misalignment.

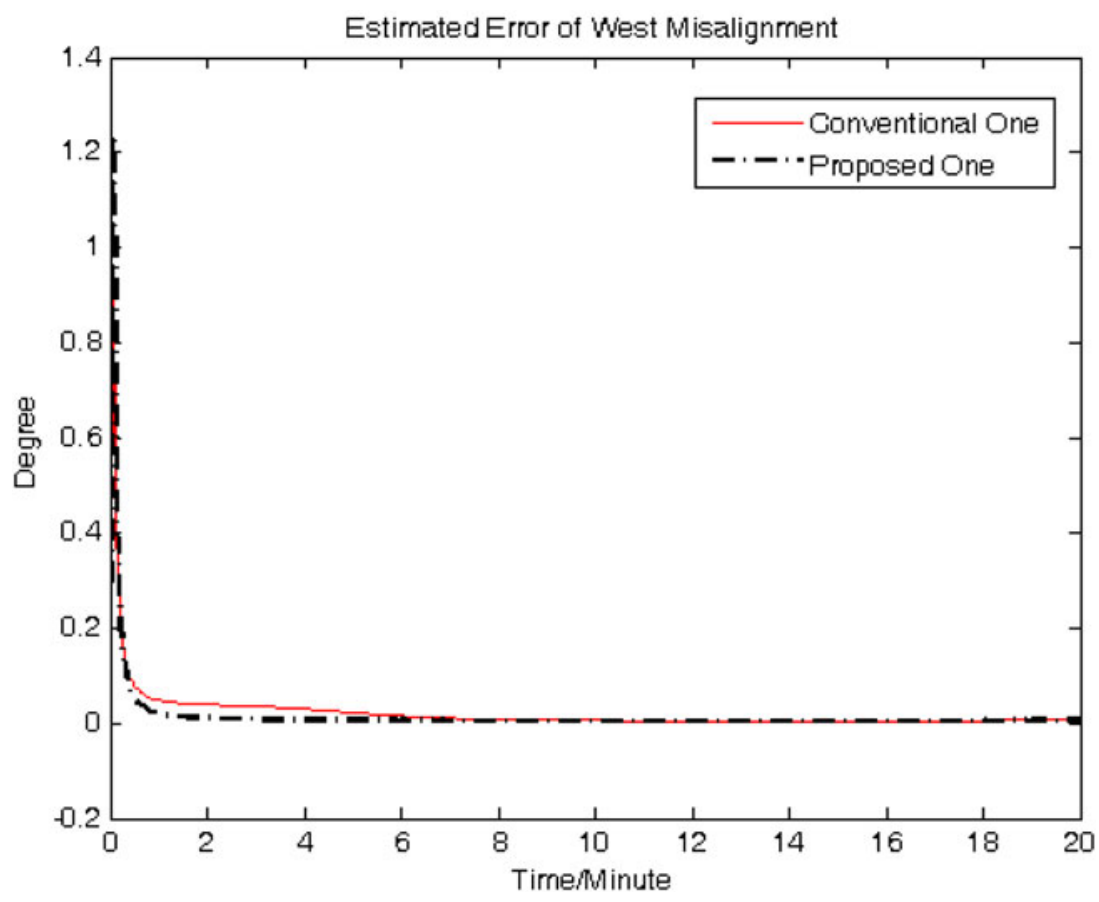

Figure 2. Estimated error of West misalignment. 


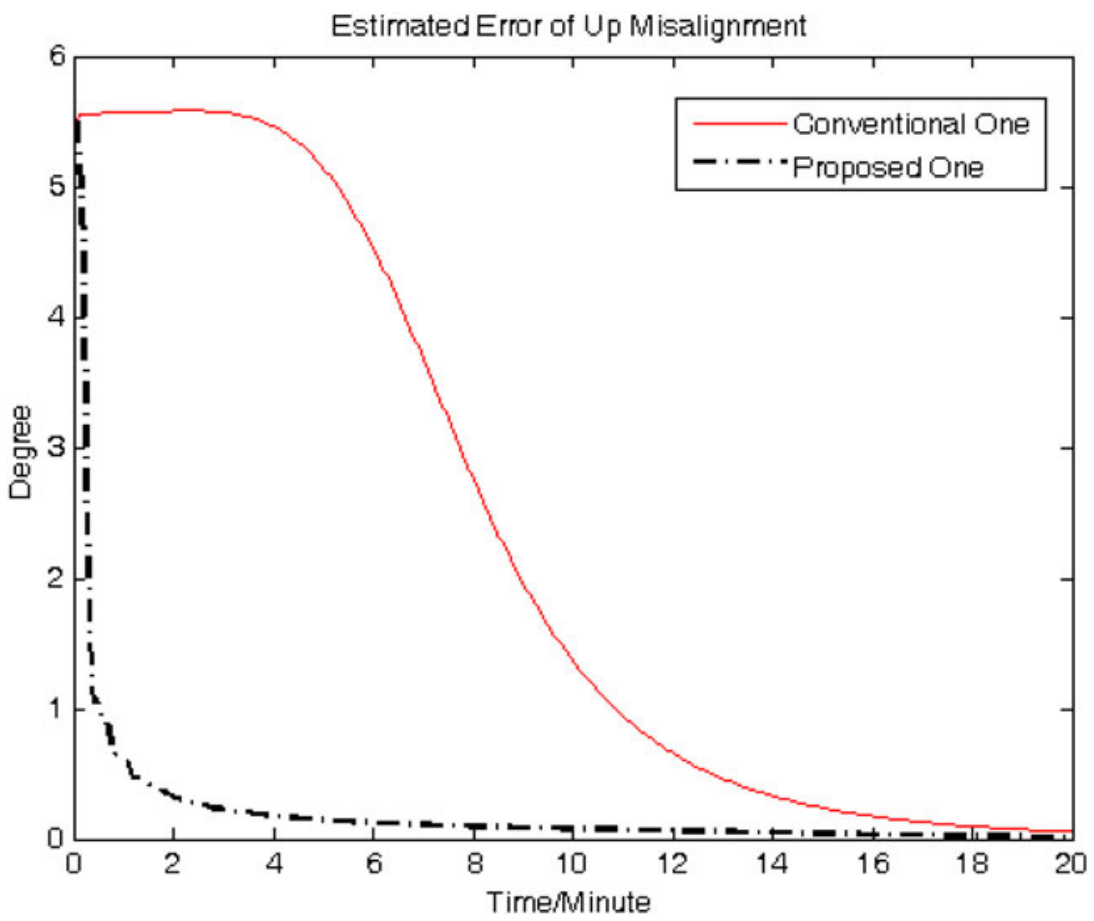

Figure 3. Estimated error of Up misalignment.

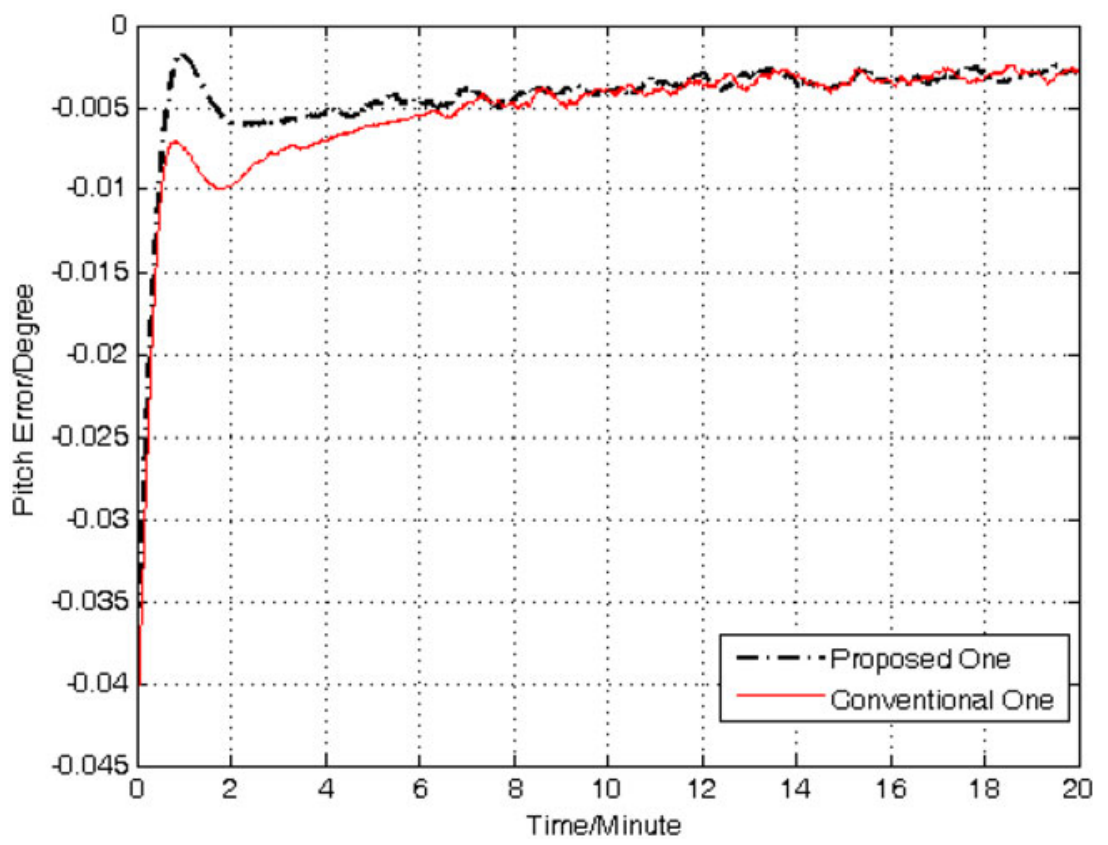

Figure 4. Pitch error of filter estimation. 


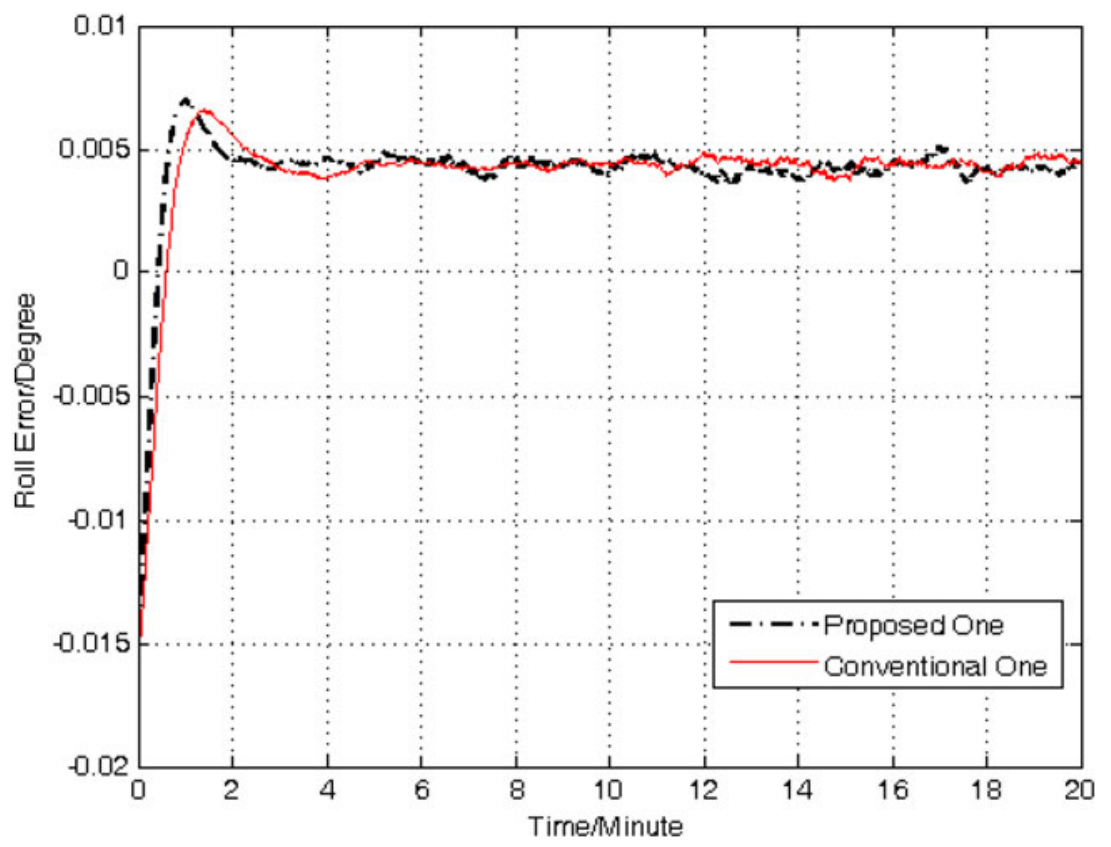

Figure 5. Roll error of filter estimation.

The lineal vibration amplitudes are:

$$
A_{D E}=0.002 \mathrm{~m} \quad A_{D N}=0.001 \mathrm{~m} \quad A_{D U}=0.003 \mathrm{~m}
$$

and:

$$
T_{D E}=T_{D N}=T_{D U}=\frac{1}{15} \mathrm{~s}
$$

Under the vibrating condition described above, simulated gyro and accelerator outputs are saved for the purpose of comparing the proposed fine alignment method with conventional one presented in Section 3. The total duration is $1200 \mathrm{~s}$. The sample frequency is chosen as $200 \mathrm{~Hz}$. The estimation processes for the misalignments are shown in Figures 1, 2 and 3. In Figures 1, 2 and 3, the y-axis depicts the misalignment estimation errors and the $\mathrm{x}$-axis depicts the time for fine alignments.

Note that we get the real-time estimations of $\alpha_{1 N}, \alpha_{1 E}$ during the process of our proposed method. Then according to Equation (47), the estimations of misalignments can be drawn in Figures 1, 2 and 3. It is clear to see in these Figures that the convergent duration for Up misalignment is longer than the other ones. Its convergent estimation value can be obtained after 10 minutes for proposed ground fine alignment, and at least 20 minutes for conventional one.

6.2. Experiment. The trial area used in this experiment was in Harbin Engineering University. The trial data was collected with the Fiber Optic Gyro (FOG) INS made by Harbin Engineering University, and the integrated navigation system composed of yhe high accuracy INS and GG24 GPS receiver. The bias stability 


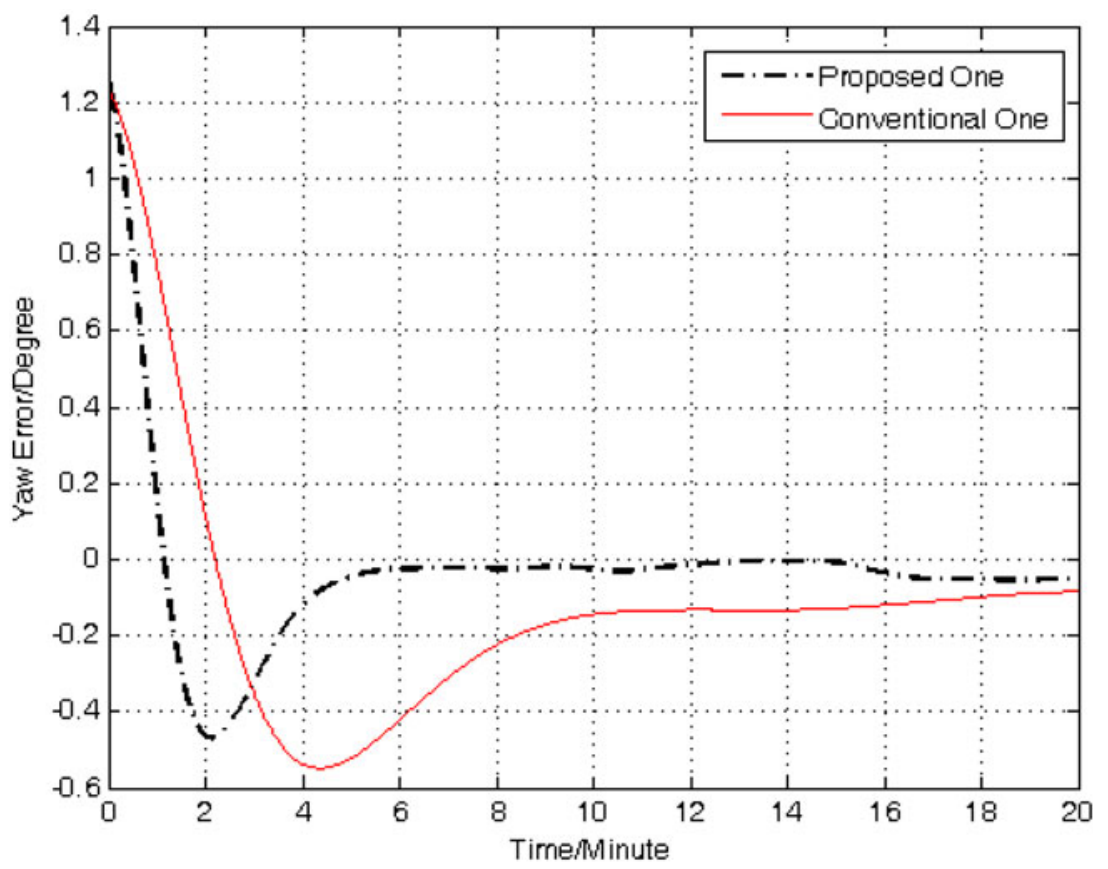

Figure 6. Yaw error of filter estimation.

of our FOG is better than $0.01 \mathrm{deg} / \mathrm{h}$. The integrated navigation system was used to generate a precise reference solution. Our FOG INS and the integrated navigation system were fixed on a board in the vehicle.

After turning on the engine, the vehicle experienced linear and angular vibrations made by the engine running, but didn't move. The trial had a 5-minute warm up period for coarse alignment. Then the trial data was collected for off-line analysis and comparison between the conventional ground fine alignment and proposed method. The off-line analysis and comparative results are shown in Figures 4, 5 and 6.

From Figures 4, 5 and 6, we can see similar results to the simulation, showing that the proposed ground alignment converges more rapidly than the conventional method.

7. CONCLUSIONS. When ground vehicles are under a vibrating base, a strapdown INS would experience angular and linear vibrations. Under that condition, getting the initial strapdown attitude matrix accurately and quickly becomes a difficult mission. In order to solve this problem, we propose a new ground fine alignment. This method uses the reconstructed error equations for strapdown INS rather than conventional one. The effects of angular and linear vibrations are considered as system and measurement noises, and don't exist in the system and measurement matrix. So utilizing the proposed method, we can accomplish the ground fine alignment under the vibrating base accurately and quickly. 


\section{REFERENCES}

Burak, H. K., Ismet, E. and Aydan, M. E. (2004). GPS/INS Enhancement for Land Navigation Using Neural Network. The Journal of Navigation, 57, 297-310.

Dai, H. D., Dai, S. W. and Ma, Z.X. (2011). Velocity Plus Partial Rate Matching Rapid Transfer Alignment. Consumer Electronics, Communications and Networks, International Conference, Xianning, Hubei Province, China.

Fang, J. C. and Yang, S. (2011). Study on Innovation Adaptive EKF for In-Flight Alignment of Airborne POS. Instrumentation and Measurement, 60, 1378-1388.

Huang, C. M., Su, W. X., Liu, P. W. and Ma, M. L. (2010). Application of Adaptive Kalman Filter Technique in Initial Alignment of Strapdown Inertial Navigation System. Proceeding of the $29^{\text {th }}$ Chinese Control Conference, Beijing, China.

Jiang, W. F. and Yu, P. L. (1992). Error Estimation of INS Ground Alignment though Observability Analysis. IEEE Transactions on Aerospace and Electronic Systems, 28, 92-97.

Qin, Y. Y. (2005). A Clever Way of SINS Coarse Alignment despite Rocking Ship. Journal of Northwestern Polytechnichal University, 23, 681-685.

Qin, Y. Y. (2006). Inertial Navigation. Science, Inc.

Silson, P. M. G. (2011). Coarse Alignment of a Ship's Strapdown Inertial Attitude Reference System Using Velocity Loci. IEEE Transactions on Instrumentation and Measurement, 60, 1930-1941.

Song, C. L., Wu, W. S., Wang, J. H. and Long, Z. Z. (2010). Cubature Gaussian Particle Filter for Initial Alignment of Strapdown Inertial Navigation System. Pervasive Computing Signal Processing and Applications (PCSPA), First International Conference on, Beijing, China.

Sun, F. (1996). Integrated Navigation System, Harbin Engineering University.

Vasconcelos, J. F., Elkaim, G., Silvestre, C., Oliveira, P. and Cardeira, B. (2011). Geometric Approach to Strapdown Magnetometer Calibration in Sensor Frame. Aerospace and Electronic Systems, 47, 1293 1306. 